\title{
Chitinolysis by Serratiae Including Serratia liquefaciens (Enterobacter liquefaciens)
}

\author{
E. MATHEW MOLISE and CHARLES H. DRAKE
}

\begin{abstract}
Bacteriology and Public Health, Washington State University, Pullman, Washington 99163
\end{abstract}
\begin{abstract}
Forty-six strains of serratiae, including 10 strains of Serratia liquefaciens (syn.: Enterobacter liquefaciens) hydrolyzed chitin in a chitin-salts-Casamino Acids agar medium. Strains which were tested on chitin-salts agar were able to use chitin as a sole source of carbon, nitrogen, and energy. None of the other members of the family Enterobacteriaceae studied produced chitinase. The ability to hydrolyze chitin may be a useful characteristic in differentiating serratiae from other Enterobacteriaceae. However, many more strains will have to be tested for chitinase synthesis before the usefulness of the test can be determined.
\end{abstract}

The genus Serratia Bizio was placed by Ewing (4) in the tribe Klebsielleae Trevisan on the basis of similarities to Klebsiella Trevisan, Enterobacter Hormaeche and Edwards, and Pectobacterium Waldee. The species in this tribe share a number of properties and are often separated on the basis of only a few characteristics. The pectobacteria are exceptional because they produce a number of extracellular enzymes which degrade pectins (10). Most pectobacteria are associated with a soil or water habitat. Colwell and Mandel (2) examined serratiae by numerical analysis and concluded that $S$. kiliersis (Lehmann and Neumann) Bergey et al., S. indica Bergey, S. plymuthica (Lehmann and Neumann) Bergey et al., and $S$. marinorubra ZoBell and Upham were not separable from $S$. marcescens Bizio. Recently, Grimont and Dulong de Rosnay (7) suggested that the serratiae are divisible into three phenons; however one of the strains studied fell outside of all three phenons.

Close similarities between $S$. marcescens and E. liquefaciens (Grimes and Hennerty) Ewing have been observed (3-7). Attempts to separate $E$. liquefaciens from serratiae have generally involved only a limited number of characteristics (8). Recently, Bascomb et al. (1), on the basis of a computer analysis of organisms in the tribe Klebsielleae, transferred $E$. liquefaciens to Serratia as $S$. liquefaciens. This transfer has been supported by Ewing et al. (5).

Monreal and Reese (9) studied chitinase production by a number of bacteria and fungi and found that $S$. marcescens QM B1466 and $E$. liquefaciens CDC 2149-57 were able to degrade chitin, the polymer of $N$-acetyl-glucosamine (linked $\beta 1-4)$. No previous studies on the Enterobacteriaceae have shown that chitinase production is a characteristic of any of the species in this family. We have tested the occurrence of this trait in 46 serratiae, including 10 strains of $S$. liquefaciens, as well as in other Enterobacteriaceae.

The serratiae tested failed to ferment lactose or arabinose but produced gelatinase, lysine and ornithine decarboxylases, extracellular deoxyribonuclease, and varied in pigment (prodigiosin) production. The strains of $S$. liquefaciens lacked pigment and fermented arabinose.

The media used to test chitinase production were chitin-salts agar (ChSA) composed of: dispersed chitin, $0.2 \% ; \mathrm{K}_{2} \mathrm{HPO}_{4}, 2 \mathrm{mM}$; $\mathrm{MgSO}_{4} \cdot 7 \quad \mathrm{H}_{2} \mathrm{O}, 2 \mu \mathrm{M}$; purified agar (Difco), $1.5 \% ; \mathrm{pH}, 7.5$ before autoclaving; and ChSA containing $0.5 \%$ vitamin-free Casamino Acids (ChSACAA) (Difco), pH, adjusted with $\mathrm{NaOH}$, as above. Finely dispersed chitin was prepared by adding $25 \mathrm{~g}$ of chitin flakes (Eastman catalog no. P2064) to 0.5 liter of cold $9 \mathrm{M}$ $\mathrm{H}_{2} \mathrm{SO}_{4}$. The slurry was allowed to hydrolyze over a 13- to 15-h period and then was diluted 12 -fold in distilled water. The fine precipitate was collected and washed repeatedly until the $\mathrm{pH}$ rose to 5. A stock suspension of $4 \%$ chitin (weight/volume) was kept refrigerated or frozen. Chitinolysis was read as a clear zone around the colony. Cultures were obtained from our departmental culture collection, Spokane (Wash.) hospitals, W. J. Brown (Wayne State School of Medicine, Detroit, Mich.), D. Blazevic (Department of Laboratory Medicine, Univ. of Minnesota, Minn.), P. Fuchs (St. Vincent Hospital, Portland, Ore.), and B. Foster 
currently in our department (cultures from Santa Clara Valley Medical Center, San Jose, Calif.), and were examined for purity and maintained on nutrient agar. Tests were made at 24 and $30 \mathrm{C}$.

The length of time required by 30 strains of Serratia, including $S$. liquefaciens, to produce visible chitinolysis at $30 \mathrm{C}$ appears in Table 1 . Cultures were inoculated on ChSA, and the times for positive reactions were from $24 \mathrm{~h}$ to 12 days for the majority of the strains. Strain 48 did not become positive until several

TABLE 1. Chitinolysis by strains of Serratia, including $S$. liquefaciens ${ }^{a}$

\begin{tabular}{|c|c|c|}
\hline \multirow[b]{2}{*}{ Strain } & \multicolumn{2}{|c|}{$\begin{array}{l}\text { Time for chitinolysis } \\
\text { to occur on }\end{array}$} \\
\hline & ChSA & $\begin{array}{l}\text { ChSACAA } \\
\text { (h) }\end{array}$ \\
\hline \multicolumn{3}{|l|}{ Serratia } \\
\hline ATCC $14764^{b}$ & $45 \mathrm{~h}$ & 12 \\
\hline ATCC 13880 & $96 \mathrm{~h}$ & 23 \\
\hline $36^{b}$ & $45 \mathrm{~h}$ & 16 \\
\hline 132 & $96 \mathrm{~h}$ & 23 \\
\hline 18 & $72 \mathrm{~h}$ & 23 \\
\hline 63 & $72 \mathrm{~h}$ & 12 \\
\hline 161 & $120 \mathrm{~h}$ & 12 \\
\hline 127 & NTc & 16 \\
\hline $2 \mathrm{G}-12^{b}$ & NT & 23 \\
\hline QM B1466 ${ }^{b}$ & NT & 23 \\
\hline 48 & $>3 \mathrm{mo}$ & 23 \\
\hline E-16 & $24 \mathrm{n}$ & 12 \\
\hline $\mathrm{E}-41$ & $72 \mathrm{~h}$ & 23 \\
\hline Bl-1 & NT & 12 \\
\hline BI-6 & $\mathrm{NT}$ & 8 \\
\hline $\mathrm{Fu}-1$ & $66 \mathrm{~h}$ & 24 \\
\hline D-1 & $288 \mathrm{~h}$ & 22 \\
\hline D-3 & $66 \mathrm{~h}$ & 20 \\
\hline CDC 862-57 & $17 \mathrm{~h}$ & 17 \\
\hline CDC 863-57 & NT & 24 \\
\hline \multicolumn{3}{|l|}{ S. liquefaciens } \\
\hline ATCC 14460 & $192 \mathrm{~h}$ & 72 \\
\hline CDC $1798-69$ & NT & 8 \\
\hline CDC $2330-68$ & NT & $168^{d}$ \\
\hline CDC $449-57$ & NT & 40 \\
\hline CDC 2144-57 & NT & 17 \\
\hline CDC 4733-57 & NT & 40 \\
\hline CDC 4637-58 & NT & 88 \\
\hline CDC $1867-59$ & NT & 24 \\
\hline CDC $1361-60$ & NT & 40 \\
\hline $\mathrm{Fu}-3$ & NT & 46 \\
\hline
\end{tabular}

a Abbreviations: ATCC, American Type Culture Collection, Rockville, Md. (cultures used not received directly from ATCC). CDC, Center for Disease Control, Atlanta, Ga., QM, Quartermaster Collection, Natick, Mass., ChSA, Chitin salts agar. ChSACAA, ChSA plus $0.5 \%$ Casamino Acids.

$b$ Pigmented strain.

c NT, not tested or no results available.

$d$ Incubated at $24 \mathrm{C}$. months. The addition of $0.5 \%$ Casamino Acids to ChSA (ChSACAA) enhanced the rate of chitinolysis such that all serratiae became positive in 8 to $24 \mathrm{~h}$, including strain 48 . Another 16 strains of serratiae not listed in Table 1 were positive within $24 \mathrm{~h}$ on ChSACAA. Nine $S$. liquefaciens strains became positive on ChSACAA in 8 to $88 \mathrm{~h}$, whereas another strain required 7 days at $24 \mathrm{C}$. Nine strains of Klebsiella pneumoniae, one of Proteus vulgaris, one of $P$. mirabilis, two of $P$. morganii, four of Enterobacter cloacae, three of Enterobacter aerogenes, one of Enterobacter alvei, two of Escherichia coli, one of Providencia sp., and one of Citrobacter sp. did not hydrolyze chitin under any condition even after several months of incubation.

Chitinolysis is not a character which has heretofore been associated with the Enterobacteriaceae. The ability of 46 strains of Serratia from diverse sources to utilize chitin suggests that this characteristic may be common to this taxon. Moreover, the ability to hydrolyze chitin is another property which supports the transfer of E. liquefaciens to Serratia. The test for chitinolysis should be added to those used to screen for Enterobacteriaceae. Because the test was negative for the other taxa studied, it may be useful in identifying nonpigmented serratiae and in differentiating them easily from other members of the family Enterobacteriaceae. A large number of strains must be examined in order to determine the value of the test. Since chitin is a substance found in arthropods and fungi which carry out their life cycles mainly in soil and water, it is suggested that the serratiae may have originated in a soil or water habitat.

\section{REPRINT REQUESTS}

Address requests for reprints to: E. Mathew Molise, Bacteriology and Public Health, Washington State University, Pullman, Wash. 99163.

\section{LITERATURE CITED}

1. Bascomb, S., S. P. Lapage, W. R. Willcox, and M. A. Curtis. 1971. Numerical classification of the tribe Klebsielleae. J. Gen. Microbiol. 66:279-295.

2. Colwell, R. R., and M. Mandel. 1965. Adansonian analysis and deoxyribonucleic acid base composition of Serratia marcescens. J. Bacteriol. 89:454461.

3. Durand, A. M., and D. J. Blazevic. 1970. Differentiation of Serratia and Enterobacter on the basis of nucleoside phosphotransferase production. Appl. Microbiol. 19:134-137. 
4. Ewing, W. H. 1972. Identification of Enterobacteriaceae, 3rd ed., Burgess Publishing Co., Minneapolis.

5. Ewing, W. H., B. R. Davis, M. A. Fife, and E. F. Lessel. 1973. Biochemical characterization of Serratia liquefaciens (Grimes and Hennerty) Bascomb et al. (formerly Enterobacter liquefaciens) and Serratia rubidaea (Stapp) comb. nov. and designation of type and neotype strains. Int. J. Syst. Bacteriol. 23:217-225.

6. Greenup, P., and D. J. Blazevic. 1971. Antibiotic susceptibilities of Serratia marcescens and Enterobacter liquefaciens. Appl. Microbiol. 22:309-314.
7. Grimont, P. A. D., and H. L. C. Dulong de Rosnay. 1972. Numerical study of 60 strains of Serratia. J. Gen. Microbiol. 72:259-268.

8. Hamilton, R. L., and W. J. Brown. 1972. Medium to aid identification of Serratia marcescens. Amer. J. Med. Technol. 38:73-76.

9. Monreal, J., and E. T. Reese. 1969. The chitinase of Serratia marcescens. Can. J. Microbiol. 15:689696.

10. Zucker, M., and L. Hankin. 1970. Regulation of pectate lyase synthesis in Pseudomonas fluorescens and Erwinia carotovora. J. Bacteriol. 104:13-18. 